\title{
An 82-year-old man with ataxia and dysarthria
}

\author{
Lilian L.Y. Vivas MD, Wayne L. Gold MD, Daniel M. Mandell MD, Peter E. Wu MD
}

A n 82-year-old man presented to the emergency department with a twoweek history of imbalance and gait disturbance resulting in a fall 10 days before presentation. There was no head injury. He previously walked without restriction, but on presentation he required a two-wheeled walker to maintain balance. He also reported a two-day history of dysarthria.

Eleven weeks prior, he was diagnosed with an infected ulcer overlying the plantar aspect of his right first metatarsal head. He had a history of type 2 diabetes mellitus and peripheral arterial disease. Treatment was started with ceftriaxone $1 \mathrm{~g}$ intravenously every 24 hours and metronidazole $500 \mathrm{mg}$ orally every 12 hours.

Other medical history included coronary artery disease, congestive heart failure, atrial fibrillation, stage 3 chronic kidney disease (estimated glomerular filtration rate $45 \mathrm{~mL} / \mathrm{min}$ ), hypothyroidism, anxiety and gout. He did not smoke or consume alcohol. Medications on presentation are listed in Box 1.

On examination, he was afebrile with a blood pressure of 147/83 $\mathrm{mm} \mathrm{Hg}$ and a heart rate of 66 beats/min that was irregularly irregular. Cardiovascular examination was otherwise normal. He had normal respiratory and abdominal examinations. Peripheral vascular examination revealed a noninfected, healing ulcer on the plantar aspect of his right foot. On neurologic examination, there was no nuchal rigidity. His mental status was normal. On language function, there was no evidence of word-finding difficulty and comprehension was normal. Cranial nerve examination revealed dysarthric speech. Articulation was impaired and his consonants were dulled. Motor examination was normal with the exception of reduced ankle reflexes (1+) bilaterally. Sensory examination revealed decreased light touch and pinprick to the midcalves as well as diminished vibration and proprioception. He had an unsteady, wide-based gait requiring a two-person assist to prevent him from falling. There was no dysdiadochokinesia or dysmetria.
Complete blood count and electrolyte measurements were normal. His serum creatinine level was 141 (normal 64-110) $\mu \mathrm{mol} / \mathrm{L}$, hemoglobin $\mathrm{A}_{1 \mathrm{C}}$ level was $8.7 \%$ and international normalized ratio was 2.19. Electrocardiography showed atrial fibrillation and a right bundle branch block, which was unchanged from previous recordings. Nonenhanced computed tomography (CT) of the brain showed chronic ischemic changes in the cerebral white matter without evidence of acute intracranial abnormalities.

\begin{tabular}{|l|l|}
\hline $\begin{array}{l}\text { Box 1: List of patient medications on } \\
\text { presentation to the emergency department }\end{array}$ \\
\hline Medication & \multicolumn{1}{c|}{ Dosage } \\
\hline $\begin{array}{l}\text { Insulin lispro } \\
\text { protamine/insulin } \\
\text { lispro }\end{array}$ & $\begin{array}{l}75 \% / 25 \% \text { mixture } \\
\text { subcutaneously twice } \\
\text { daily }\end{array}$ \\
\hline Amlodipine & $5 \mathrm{mg}$ orally daily \\
\hline Candesartan & $4 \mathrm{mg}$ orally daily \\
\hline Hydralazine & $5 \mathrm{mg}$ orally twice daily \\
\hline Metoprolol & $\begin{array}{l}12.5 \mathrm{mg} \text { orally twice } \\
\text { daily }\end{array}$ \\
\hline Warfarin & $5 \mathrm{mg}$ orally daily \\
\hline Ezetimibe & $10 \mathrm{mg}$ orally daily \\
\hline Omeprazole & $20 \mathrm{mg}$ orally daily \\
\hline Levothyroxine & $0.1 \mathrm{mg}$ orally daily \\
\hline Sertraline & $100 \mathrm{mg}$ orally daily \\
\hline Ceftriaxone & $1 \mathrm{~g}$ intravenously daily \\
\hline Metronidazole & $\begin{array}{l}500 \mathrm{mg} \text { orally twice } \\
\text { daily }\end{array}$ \\
\hline
\end{tabular}

Competing interests: None declared.

This article has been peer reviewed.

The authors have obtained patient consent.

Correspondence to:

PeterWu, Pete.Wu@mail. utoronto.ca

CMAJ 2016. DOI:10.1503 /cmaj.150573

\footnotetext{
- Ker POINTS

- Metronidazole-induced encephalopathy is a rare idiosyncratic reaction.

- Most cases resolve with discontinuation of metronidazole.

- Idiosyncratic drug reactions are unpredictable and difficult to diagnose.

- New and unexplained symptoms following treatment with a new medication merit consideration of an adverse reaction.

- Identifying adverse drug reactions will help to mitigate consequences and prevent re-exposure.
} 
Given his presentation of subacute ataxia and dysarthria with nondiagnostic CT, the patient was admitted to hospital for further investigation and management.

\section{What are the next steps?}

a. Lumbar puncture with cerebrospinal fluid analysis

b. Electromyography and nerve conduction studies

c. Magnetic resonance imaging (MRI) of the brain

d. Supportive physical and occupational therapy

The correct answers are (c) MRI of the brain and (d) supportive physical and occupational therapy. This patient's signs and symptoms localize to the posterior circulation and cerebellum. Compared with CT, MRI provides greater detail for examination of the structures of the posterior fossa, and characterizing pathology in this territory would be the most appropriate next step.

The MRI revealed bilaterally symmetric hyperintensity in the dentate nuclei of the cerebellum and in the splenium of the corpus callosum on $T_{2}$-weighted fluid-attenuated inversion recovery (FLAIR) images (Figure 1). There were also chronic ischemic changes in the periventricular and deep white matter of the cerebral hemispheres as previously shown on CT, a common finding related to advanced age and vascular risk factors.

In consideration of his functional impairment and falls, supportive physical and occupational therapy (d) was also initiated to aid in his daily activities. Lumbar puncture and cerebrospinal fluid analysis (a) was not performed on presentation because the patient was afebrile and not immunocompromised, lowering the suspicion for infection. Although infections of the central nervous system can present with cerebellar symptoms and without fever, a lumbar puncture was not chosen as one of the immediate next steps but would be part of a second tier of investigations if the MRI were nondiagnostic. Lastly, although there was evidence of diabetic sensory neuropathy on examination, electromyography and nerve conduction studies (b) would not assist in the diagnosis of his dysarthria.

\section{Based on the findings on MRI, what is the most likely diagnosis?}
a. Multiple sclerosis
b. Infectious encephalopathy
c. Toxic encephalopathy
d. Alzheimer dementia

The cerebellar abnormalities on MRI conform to the dentate nuclei rather than a particular vascular territory, which argues against infarction as a cause. These MRI findings are inconsistent with multiple sclerosis (a), because demyelination tends to preferentially affect white matter more than grey matter (nuclei). The symmetry of the imaging findings, as well as involvement of the dentate nuclei and splenium of the corpus callosum, suggests either a metabolic, toxic, infectious or neurodegenerative process. ${ }^{1,2}$ With normal cognition, the absence of volume loss on neuroimaging and

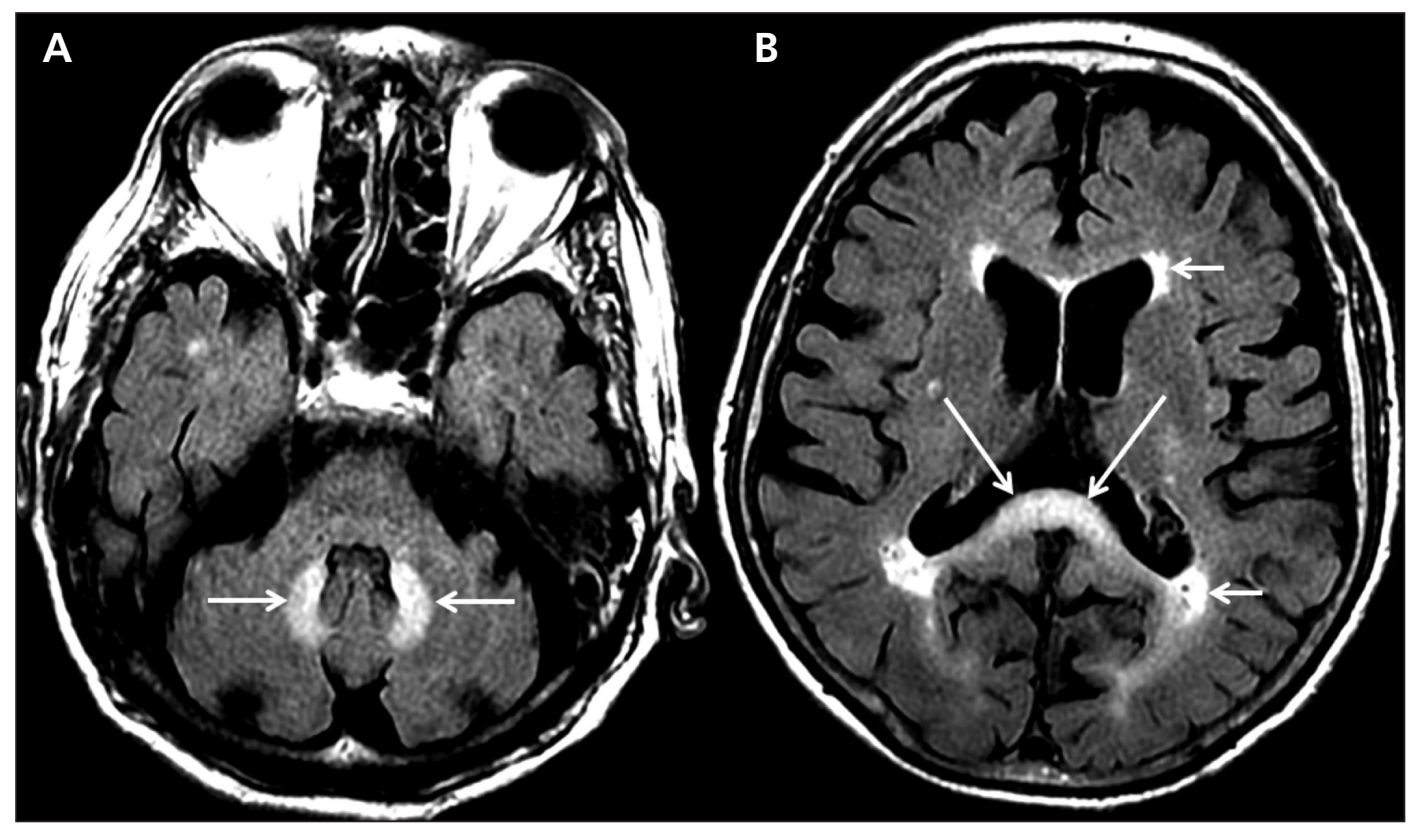

Figure 1: Axial $T_{2}$-weighted fluid-attenuated inversion recovery (FLAIR) magnetic resonance images showing (A) bilaterally symmetric hyperintensity in the dentate nuclei of the cerebellum (arrows), and (B) splenium of the corpus callosum (long arrows) and chronic ischemic changes in the cerebral white matter (short arrows). 
normal-sized ventricles, Alzheimer dementia (d) is unlikely. Infectious encephalopathy (b) is still a consideration.

Given this patient's medication exposure and MRI findings, the leading diagnosis is (c) toxic encephalopathy secondary to metronidazole, also known as metronidazole-induced encephalopathy. The most common imaging finding of metronidazole-induced encephalopathy is symmetric hyperintensity on $T_{2}$-weighted images in the dentate nuclei. ${ }^{2,3}$ The splenium of the corpus callosum, periaqueductal grey matter and dorsal brainstem are often also involved. ${ }^{3,4}$ Less commonly, there is abnormality in the cerebral hemispheric white matter or basal ganglia. ${ }^{4}$ This patient had received metronidazole for 11 weeks for his foot ulcer, with a total cumulative dose of more than $80 \mathrm{~g}$.

At the time of our assessment, his ulcer no longer appeared infected; therefore, both the ceftriaxone and the metronidazole were stopped. Seven days following the discontinuation of the metronidazole, he showed marked improvement of his cerebellar symptoms to the point that he could ambulate without assistance, and his speech had returned to normal. One month following discontinuation of his metronidazole he had complete resolution of his cerebellar signs and symptoms, supporting the diagnosis of metronidazoleinduced encephalopathy. This diagnosis is further substantiated with application of the Naranjo Adverse Drug Reaction Probability Scale. The patient scored 7 on the scale, suggesting a probable adverse drug reaction (Box 2). ${ }^{5}$

\section{Discussion}

Metronidazole is a 5-nitroimidazole antimicrobial with activity against protozoa and anaerobic bacteria. ${ }^{3}$ Reduction of metronidazole produces reactive metabolites, which result in disruption of the DNA of microbial cells, culminating in cell death. ${ }^{3}$ Although metronidazole is generally welltolerated with broad clinical utility, it is known to cause idiosyncratic neurologic reactions, specifically peripheral sensory neuropathy and encephalopathy. ${ }^{3}$ Though rare, both of these reactions can result in substantial functional impairment, and merit appropriate counselling and monitoring.

\section{Clinical features of metronidazole- induced encephalopathy}

Metronidazole-induced encephalopathy is a rare adverse drug reaction. To date, a manual literature review identified only 91 reported cases. Clinical features may include cerebellar dysfunction, altered mental status and seizures. ${ }^{4,6}$ Our patient presented with symptoms of cerebellar dysfunction. A systematic review of case reports and case series involving 64 patients showed that $75 \%$ of affected patients had cerebellar dysfunction, $33 \%$ had altered mental status and $13 \%$ had seizures; $17 \%$ of patients had both cerebellar dysfunction and altered mental status; and one

\section{Box 2: Naranjo Adverse Drug Reaction Probability Scale*†}

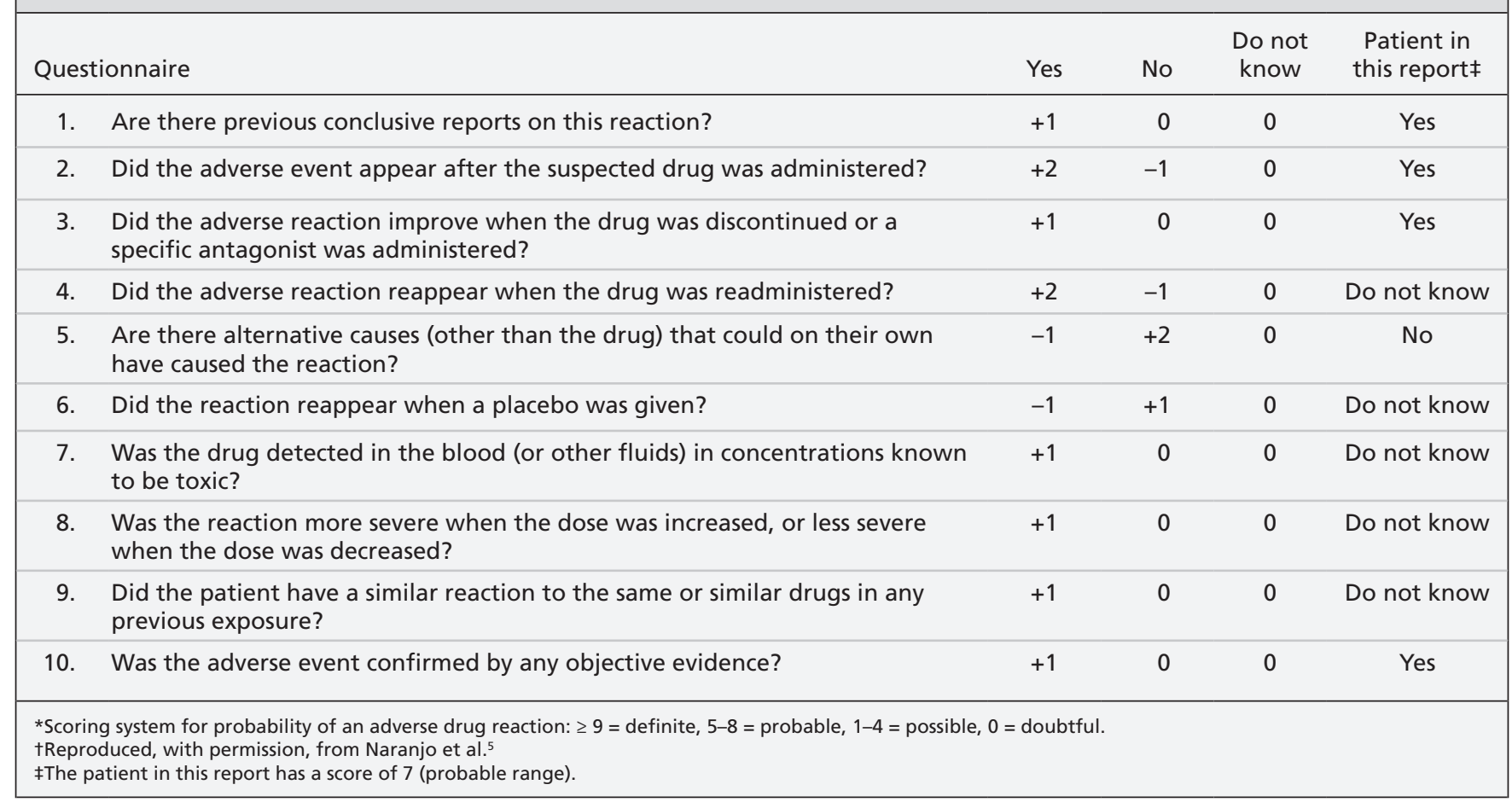


patient presented with all three manifestations. ${ }^{6}$ Cases of metronidazole-induced encephalopathy have been reported with total cumulative doses of as low as $0.25 \mathrm{~g}$ to as high as $182 \mathrm{~g}$, occurring over durations from one day to six months. ${ }^{3,6}$

\section{Pathophysiology of metronidazole- induced encephalopathy}

The mechanism of neurologic injury in metronidazole-induced encephalopathy is postulated, but is not completely understood. Metronidazole has a large volume of distribution and readily crosses the blood-brain barrier. ${ }^{3}$ Experimental models show that intermediate metabolites of metronidazole can bind neuronal RNA, resulting in reversible axonal edema, as well as possibly modulating the expression of $\gamma$-aminobutyric acid. ${ }^{3,4,6,7}$

\section{Idiosyncratic drug reactions}

In general, idiosyncratic drug reactions are unpredictable adverse effects that cannot be explained based on the pharmacology of a drug. ${ }^{8,9}$ Although the exact mechanism is still incompletely understood, these events are often believed to result from reactive metabolites of a drug and not the drug itself. ${ }^{8,9}$ They are dose-dependent reactions in individuals who are susceptible; however, there is no specific dose at which they occur in most patients. ${ }^{8}$ These reactions can affect any organ system, but commonly involve the liver, skin and bone marrow, and can be accompanied by fever. ${ }^{9}$ Because these events are idiosyncratic, monitoring for adverse reactions might not prevent onset, but will allow for earlier recognition, mitigation of consequences and prevention of re-exposure.

In addition to discontinuation of the drug, treatment is primarily supportive. This patient's metronidazole was discontinued, resulting in rapid clinical improvement. Most reported cases of metronidazole-induced encephalopathy have had good outcomes with resolution of signs, symptoms and MRI findings after drug cessation. Although the prognosis appears to be favourable overall, there are two case reports in which metronidazole-induced encephalopathy contributed to death, ${ }^{10,11}$ emphasizing the importance of timely recognition of a condition that is largely reversible.

\section{Conclusion}

This case highlights a rare adverse effect of a commonly prescribed medication. With an exposure history, adverse drug reactions should be on the differential diagnosis for any new-onset clinical signs and symptoms, but should be specifically considered when standard investigations are nondiagnostic.

\section{References}

1. Simonetta F, Christou F, Vandoni RE, et al. Walking unsteadily: a case of acute cerebellar ataxia. BMJ Case Rep Jan. 2; 2013

2. Kim E, Na DG, Kim EY, et al. MR imaging of metronidazoleinduced encephalopathy: lesion distribution and diffusionweighted imaging findings. AJNR Am J Neuroradiol 2007;28: 1652-8

3. Bottenberg MM, Hegge KA, Eastman KD, et al. Metronidazoleinduced encephalopathy: a case report and review of the literature. J Clin Pharmacol 2011;51:112-6.

4. Rimkus Cde M, Andrade CS, Leite Cda C, et al. Toxic leukoencephalopathies, including drug, medication, environmental and radiation-induced encephalopathic syndromes. Semin Ultrasound CT MR 2014;35:97-117.

5. Naranjo CA, Busto U, Seller EM, et al. A method for estimating the probability of adverse drug reactions. Clin Pharmacol Ther 1981;30:239-45

6. Kuriyama A, Jackson JL, Doi A, et al. Metronidazole-induced central nervous toxicity: a systematic review. Clin Neuropharmacol 2011;34:241-7.

7. Seok JI, Yi H, Song YM, et al. Metronidazole-induced encephalopathy and inferior olivary hypertrophy: lesion analysis with diffusion-weighted imaging and apparent diffusion coefficient maps. Arch Neurol 2003;60:1796-800.

8. Knowles SR, Uetrecht J, Shear NH. Idiosyncratic drug reactions: the reactive metabolite syndromes. Lancet 2000;356: 1587-91.

9. Uetrecht J, Naisbitt DJ. Idiosyncratic adverse drug reactions: current concepts. Pharmacol Rev 2013;65:779-808.

10. Hobbs K, Stern-Nezer S, Buckwalter MS, et al. Metronidazoleinduced encephalopathy: not always a reversible situation. Neurocrit Care 2015;22:429-36.

11. Groothoff MV, Hofmeijer J, Sikma MA, et al. Irreversible encephalopathy after treatment with high-dose intravenous metronidazole. Clin Ther 2010;32:60-4.

Affiliations: Department of Medicine (Vivas, Gold, Wu), Division of Physical Medicine and Rehabilitation (Vivas), University of Toronto; Division of Infectious Diseases (Gold), University Health Network; Department of Medical Imaging (Mandell), University of Toronto; Division of Neuroradiology (Mandell), University Health Network; Division of Clinical Pharmacology and Toxicology (Wu), University of Toronto, Toronto, Ont.

Contributors: All of the authors were involved in the clinical care of this patient. Wayne Gold and Peter Wu were involved in the conception of this report. All of the authors were involved in the literature review. Lilian Vivas and Peter Wu drafted the manuscript, which all authors revised. All of the authors gave final approval of the version to be published and agreed to act as guarantors of the work. 